\title{
A Case of Massive Recurrent Rheumatoid Effusion
}

\author{
Nagadarshini Vinod ${ }^{1}$, Hassan Tahir ${ }^{1, *}$, Arslan Ahmed ${ }^{2}$, Asad Ullah ${ }^{1}$, Medha Joshi ${ }^{1}$ \\ ${ }^{1}$ Temple University/Conemaugh Memorial Hospital Johnstown PA \\ ${ }^{2}$ Ghurki Trust Teaching Hospital Lahore Pakistan \\ *Corresponding author: Hassantahir_01@hotmail.com
}

\begin{abstract}
Rheumatoid arthritis (RA) is a well-known auto-immune disease affecting the joints, its signature signs being bone erosions and joint deformity. Other than arthritis, RA can also affect other organs in the body, such as eyes, heart, lungs, etc. In our case we are going to discuss an elderly lady with RA, presenting with large pleural effusion as presenting complaint.
\end{abstract}

Keywords: rheumatoid arthritis, pleural effusion

Cite This Article: Nagadarshini Vinod, Hassan Tahir, Arslan Ahmed, Asad Ullah, and Medha Joshi, "A Case of Massive Recurrent Rheumatoid Effusion.” American Journal of Medical Case Reports, vol. 5, no. 5 (2017): 129-130. doi: 10.12691/ajmcr-5-5-7.

\section{Introduction}

Rheumatoid arthritis (RA) is a well known systemic inflammatory disorder that affects the joints, usually associated with progressive, symmetric, erosion of cartilage and bone.Rheumatoid arthritis affects approximately $1 \%$ population of developed countries [1]. Even though joint disease is the most common presentation of RA, it can also present with extra-articular complications such as subcutaneous nodule formation, vasculitis, scleritis, uveitis and lung disease. Of all the extra-articular complications, lung disease has the highest morbidity and mortality [1] and of all the pulmonary complications, pleural disease is the most common [2]. Massive, symptomatic effusions in RA are uncommon with a prevalence of about $2 \%-5 \%$ and are usually noticed in long standing active disease [3]. In our case report, we discuss an elderly lady, with history of RA, who presented with severe dyspnea and was found to have a massive pleural effusion.

\section{Case Presentation}

A 62-year-old female with pertinent medical history of Graves' disease, untreated rheumatoid arthritis presented with cough and shortness of breath. Patient was in her usual state of health until 5 months ago, when she was diagnosed with rheumatoid arthritis.

Around the same time, patient started to notice neck spasm. She noted that she was unable to lift her chin up which later progressed to dysphagia, predominantly to solids. According to her, she had difficulty swallowing due to head and neck position. She had dry cough which was progressively getting worse in the past 2 weeks, associated with low-grade fever. Patient denied any rash. She did admit significant weight loss due to decreased appetite, approximately 40 pound weight loss over the course of 3 months.
On initial evaluation in the ER her vitals were, temperature $37.1 \mathrm{C}$, pulse $85 \mathrm{bpm}$, respiratory rate 18 , blood pressure 113/63 mm hg, and 93\% oxygen saturation on room air. Upon examination of hands she had severe swan neck deformities of both hands along with rheumatoid nodules. Lung examination revealed decreased breath sounds on left lung zones. Chest $\mathrm{x}$-ray was obtained which revealed massive left pleural effusion. In the interim, a CT scan of chest without contrast was obtained which revealed large left and trace right pleural effusion. It was also noted to have moderate pericardial effusion. She underwent echocardiogram which confirmed effusion of $1.6 \mathrm{~cm}$ without any tamponade. Patient's white blood cell count was 9,700, without anemia or thrombocytopenia, sedimentation rate was 33, C-reactive protein was 44.7 , rheumatoid factor 80 , stable kidney functions. Thoracentesis was performed and approximately 2.5 liters of fluid was drained. Pleural fluid analysis revealed $\mathrm{Ph} 8$, Lactate dehydrogenase ( $\mathrm{LDH}) 437$, protein 38 , glucose 80 , white blood cells 1520 , red blood cells 500 , neutrophils $48 \%$, lymphocytes $30 \%$, LDH serum 900 , rheumatoid factor with a titer of 1200 , bacterial cultures, gram stain negative, ADA undetectable and negative AFB staining. After thoracentesis, patient's dyspnea improved tremendously.Unfortunately, within a span of 48 hours she again developed massive fluid build up in left lung, further studies revealed lower glucose levels of 40, elevated LDH and RF titles of 1580 . She had 3 episodes of pleural effusion in a span of 30 days, for which she is currently scheduled for thoracotomy followed by decortication, in the interim she is on intravenous steroids to. Post surgery she will be transitioned to oral steroids and methotrexate for rheumatoid arthritis.

\section{Discussion}

Pleural involvement is the most common pulmonary manifestation of RA and its incidence is more common in 
middle aged males (age>35years) and those with rheumatoid nodules [1]. Occasionally pericardial effusion may co-exist with underlying pleural effusion [2]. It usually presents as small pleural effusions noted in up to $70 \%$ on autopsy studies [4,5]. Various theories behind the pathogenesis of development of pleural effusion are impaired fluid resorption from inflamed pleura, necrosis of sub-pleural rheumatoid nodules, and local production of cytokines and immune complexes accentuating endothelial injury [6].

The pathognomonic features of the exudative pleural fluid that goes in favor of diagnosis of Rheumatoid pleural effusion include low glucose levels $(<25)$, high lactate dehydrogenase (LDH) level (2-3 times upper limit of normal), high RF titers that exceed levels in the serum. In our patient most of the above features were observed except for higher ph and glucose levels. During acute onset of rheumatoid pleural effusion pleural fluid $\mathrm{Ph}$ and glucose might be equivalent to serum glucose and $\mathrm{Ph}$ making it difficult to differentiate from bacterial etiology. However lower levels $\mathrm{Ph}$ and glucose in pleural fluid is noted in subsequent effusions and in chronic pleural effusion [1] as seen in our case. Even though lymphocyte predominance in pleural fluid is expected in any autoimmune process, neutrophil predominance can also manifest in rheumatoid pleural effusion [2].

Initial treatment of pleuritis with nonsteroidal antiinflammatory agents (NSAIDS) may be sufficient. Most of the patients respond to corticosteroids, for the patients who fail to respond role of corticosteroid injections into the pleural space is questionable $[8,9]$. Occasionally, repeated, symptomatic effusions may require decortication, which is highly invasive [10].

This case describes the rare complication of untreated rheumatoid arthritis i.e. rapid recurrence of large pleural effusions resulting in multiple thoracentesis. Massive pleural effusions are relatively rare in RA, and pathognomonic features described in various literatures might not be seen in all rheumatoid pleural effusions

\section{Conclusion}

Rheumatoid effusion should be considered as a potential differential diagnosis in any case of unexplained, recurrent pleural effusion. Untreated rheumatoid arthritis can manifest as more than just joint inflammation or deformity, such as massive pleural effusion in our case. Not all rheumatoid effusions will have hallmark features as mentioned in many literatures, as in our case which presented with high $\mathrm{Ph}$ and glucose during initial evaluation, which can be misdiagnosed as bacterial infection.

\section{References}

[1] Rheumatoid arthritis-associated lung disease Megan Shaw, Bridget F. Collins, Lawrence A. Ho, Ganesh Raghu European Respiratory Review 2015 24: 1-16.

[2] Massive Pleural Effusion: A Rare Presentation of Rheumatoid Arthritis*Steve Egwuonwu.

[3] Walker W, Wright V. Rheumatoid pleuritis. Ann Rheum Dis. 1967; 26(6): 467-474.

[4] Corcoran JP, Ahmad M, Mukherjee R, et al. Pleuro-pulmonary complications of rheumatoid arthritis. Respir Care 2014; 59: e55-e59.

[5] Case records of the Massachusetts General Hospital. Weekly clinicopathological exercises. Case 8-2002. A 56-year-old woman with a persistent left-sided pleural effusion. N Engl J Med 2002; 346: 843-850

[6] Balbir-Gurman A, Yigla M, Nahir AM, et al. Rheumatoid pleural effusion. Semin Arthritis Rheum 2006; 35: 368-378.

[7] Demosthenes B, Ioannis P, Argyris T. Pleural Involvement in Systemic Autoimmune Disorders. Respiration 2008.

[8] Bouros D., Pneumatikos I., Tzouvelekis A. pleural involvement in systemic autoimmune disorders. Respiration. 2008; 75: 361-371.

[9] Rheumatoid pleural effusion presenting as pseudochylothorax in a patient without previous diagnosis of rheumatoid arthritis Tetsuya Yokosuka, a,* Asako Suda,b Midori Sugisaki,b Manabu Suzuki,b Ritsuko Narato,b Hitoshi Saito,b Tatsuji Enomoto,b Toshiko Kobayashi,a and Koichiro Nomurab.

[10] Yarbrough J.W., Sealy W.C., Miller J.A. Thoracic surgical problems associated with rheumatoid arthritis. J Thorac Cardiovasc Surg. 1975; 69: 347-354. 